\section{Leading innovation and improvement}

\section{MEDICAL GRAND ROUNDS - ASSESSING THE IMPACT AND IMPROVING ATTENDANCE}

Helena Edwards*, Amanda Laird, Zoe Burton, Alex Hobson. Portsmouth Hospitals NHS Trust

\subsection{6/leader-2018-FMLM.95}

Introduction Medical grand rounds are a well-established form of postgraduate medical education and are still a component of most UK hospital teaching programmes. Our large acute district general hospital runs a weekly grand round, which is open to all clinicians.

Aims Attendance at grand round has gradually reduced over the preceding years in our hospital, which is also reflected nationally. The aim of this project was to assess the reasons for the decline in attendance and then implement changes that led to a sustained improvement.

Methods A 10-item questionnaire was developed to assess whether grand round still provided an effective learning opportunity and to identify factors affecting attendance. There were 98 respondents from a variety of medical grades. The majority of respondents did not attend grand round on a regular basis, however there was enthusiasm for grand round with $68 \%$ of respondents saying that it provided an effective learning environment.

A number of factors affecting attendance were identified including workload and clinical commitments, logistical issues such as inconsistent venue and lack of refreshments, and culture. These were addressed with a number of interventions such as posters for advertising, drug rep sponsorship, the use of high profile speakers and attempts to improve the culture.

Results The improvement strategy has been a continuous project over the last year from September 2017. Attendance of grand round has been monitored using a sign-in register for each session. Written feedback was also collected from each session to allow us to develop future programmes. The results have shown a gradual, but consistent increase in attendance at grand round from an average of 16 attendees to 36 .

\section{Leading innovation and improvement}

\section{LEADING IN COMMUNICATION BETWEEN HEALTHCARE PROFESSIONALS}

${ }^{1}$ Fatemeh Keshtkar*, ${ }^{2}$ Bill Campbell. ' Royal Victoria Hospital, UK; ${ }^{2}$ Causeway Hospital, UK

10.1136/leader-2018-FMLM.96

The aim of this project was to assess effectiveness of communication among health care professionals, with view to improving handover, team work and patient safety.

Background Communication between the multidisciplinary team and handover is a perilous procedures in medicine, and when carried out improperly can be a major contributory factor to subsequent error and harm to patients. Within the surgical departments in Causeway Hospital an increasing number of adverse incidents were noted due to poor communication between healthcare professionals

Methodology A questionnaire was distributed among members of frontline staff. The questionnaire was designed to assess how well members felt information was communicated. The quality and content of written communication was also assessed to identify pit falls. Recorded adverse incidents with root cause analysis leading to communication issues were also taken into account.

As a result of the problem areas identified in written communication, a proforma was made for purposes of handover. A lead was assigned in order to evaluate methods of handover, to audit this and make changes where required. Staff were trained in human factors and SBAR. A lead communication nurse was selected to ensure appropriate use of the proforma and training staff.

Results A further questionnaire was distributed following the implemented changes-staff felt communication had significantly improved and there were fewer errors as a result.

Analysis of written notes showed the use of post it notes had significantly reduced (to less than half) and there was $100 \%$ compliance with use of proforma which were correctly filled in $100 \%$ of cases.

Following implemented changes there were no recorded adverse incidents as a result of poor communication among multidisciplinary team.

Conclusion Assigning and training leaders in communication among front line staff can improve perceptions and reduce adverse events as a result of poor communication.

\section{Education, leadership, QI project}

\section{BRINGING THE CURRICULUM TO LIFE}

${ }^{1}$ Akudo Okereafor*, 'Oliver Campbell, 'Ashley Ong, 'Hannah Jacob, 'Jenny Pallawela, ${ }^{1}$ Vicky Jones, ${ }^{2}$ Nicola Davey. ${ }^{1}$ North Middlesex University Hospital; ${ }^{2}$ Quality Improvement Clinic

10.1136/leader-2018-FMLM.97

Problem Lack of knowledge about the new RCPCH curriculum suggested trainees would be unprepared for the new professional requirements.

Aim All paediatric trainees and trainers to be aware and feel confident to use the new curriculum.

Measures Awareness, Confidence

Change ideas Focused on identifying learning in everyday experiences.

PDSA\# 1: Face-to-face presentations.

PDSA\#2: Night teams highlight an issue at Monday handover using the 'Learning Point from Our Nights' proforma e. g.unusual cases. Team agreement on learning point mapped to new curriculum, proformas shared via WhatsApp.

PDSA\#3: Short videos created weekly with learning points

PDSA\#4: Focus on mandatory elements of curriculum.

PDSA\#5: Online learning platform to catalogue curriculum learning tools.

PDSA\# 6: Sharing with other paediatric departments.

Results Baseline data: 0/27 trainees/trainers felt confident to start using the new RCPCH curriculum.Following a series of interventions there was substantial progress in curriculum knowledge: Those responding 'I've heard of it' fell by 19 . 'I've read about it and know it' rose by 10 . 'I feel confident to start using it in August' increased from zero to nine.

\section{Conclusions}

- Driver: a need to understand and know how to use the new $\mathrm{RCPCH}$ Progress curriculum ahead of its launch in August. 
- Change ideas mapped to the new curriculum helped paediatric trainees and consultants increase their curriculum knowledge and confidence.

- Developing a habit of reflection after night shifts facilitates discussions about decision-making and autonomy, identification of real-time problems and promotes team feedback and cohesion .

- Disseminating completed templates and bite-sized learning videos via WhatsApp and uploading them to a Trello learning platform gave benefit regardless of shift-pattern.

- Collaborative learning increased our departments' collective knowledge, encouraged evidence-based learning, improved our professional development and will ultimately enrich our patient care.

\section{Leadership}

\section{INSPIRING JUNIOR DOCTORS INTO LEADERSHIP}

Katie Wallace*, Sara Page, Alexandra Hughes, Jahangir Ahmed. Sheffield Teaching Hospitals, UK

\subsection{6/leader-2018-FMLM.98}

Many junior doctors are unsure how to engage with leadership and feel that it is a gap in their training. Meanwhile senior colleagues are trying to find ways to improve junior doctor morale and thereby recruitment and retention.

Leadership connections was founded as a way of enabling and empowering leadership development among junior doctors. The structure is simple; a monthly seminar delivered by different people on different topics each month but with a common theme of leadership. The aim is to create a supportive community therefore attendees leave with not only a skill and an attendance certificate but know that the fixed times and location is something they can return to again in the future.

Yorkshire and Humber is home to the excellent Future leaders programme but having completed a 12 month leadership fellowship many found themselves lost on returning to work therefore this format allows those people to continue their leadership journey and an opportunity to share their work.

Leadership connections was founded and run by junior doctors with a collaboration across specialities and grades. In establishing this group individuals have had the opportunity to demonstrate skills in negotiation, planning, bidding for funding and communication. We have been able to connect those that attend with further development opportunities for example participating in local trust committees.

Attendance is doubling with each event and feedback is positive. We hope that through inspiring wider participation and engagement among junior doctors we can influence the wider culture and improve poor morale.
NHS, workforce, prevention, empowerment

\section{SURGICALLY FIXING THE NHS: VALUING PEOPLE, EXERCISE AND SKILLS}

Scarlett McNally*. East Sussex Healthcare NHS Trust, UK

10.1136/leader-2018-FMLM.99

The NHS is failing. There are increasing numbers of older patients with multiple co-morbidities, including frailty and dementia. $70 \%$ of NHS spend is on preventable conditions. Yet health is still focussed on interventions and passive care rather than facilitating exercise, nutrition, environment and culture. ${ }^{1}$ Similarly, healthcare leadership suffers with financial constraints, burdensome targets and overwhelming workload. NHS staff feel undervalued with burnout, low morale, inadvertent bullying or being set up to fail (Glass Cliff phenomenon). Perhaps the current paradigms could be challenged?

A new approach is needed, focussing on:

- Clear values, to get people through tough days

- Knowledge about how health really works including primary and secondary prevention and iatrogenic illness

- Skills: Understanding that good 'leadership' requires 'management' skills, involving people, change, projects, finance, education, IT and communication.

- Aiming for precision and clarity:

- Being clear about expectations helps reduce bullying

- Having defined job roles reduces unconscious bias

- Identifying what is not possible

- Empowering and valuing all individuals

- Ensuring that highly skilled workers (eg doctors) can focus on decision-making and handling uncertainty, with some other tasks delegated to 'Doctors' Assistants'

- Using team-working and the community to support anyone having a 'below average' day

- Getting all NHS staff to work on the prevention agenda, advocating environments, expectations and practicalities to improve health. ${ }^{1} 41 \%$ of over- 65 s are classified as 'inactive'. Lack of fitness is reversible and reduces their need for social care. ${ }^{3}$ Health workers are ideally placed to work on goals, habits and practicalities with individuals, communities and wider organisations (including education, transport and sport).

- All management decisions should satisfy the bigger picture as well as the detail (eg building cycle parking and having flexible working policies)

\section{REFERENCES}

1. Exercise: the miracle cure (2015, Academy). www.aomrc.org.uk/publications/ reports-guidance/exercise-the-miracle-cure-0215

2. Doctors' Assistants. https://healthmanagement.org/c/healthmanagement/ issuearticle/award-winning-new-doctors-assistants-freeing-time-in-acute-nhshospitals

3. McNally, et al. Focus on physical activity can help avoid unnecessary social care. BMJ 2017;359:j4609. https://doi.org/10.1136/bmj.j4609 or on www.scarlettmcnally.co.uk 